\title{
Enhanced Reduction of Thermal Conductivity in Amorphous Silicon Nitride Containing Phononic Crystals Fabricated Using Directed Self-Assembly of Block Copolymers
}

Chun Zhou, ${ }^{\dagger}$ Naoki Tambo, ${ }^{\ddagger}, *$ Elizabeth Michiko Ashley, ${ }^{\dagger}$ Yuxuan Liao, ${ }^{\S}$ Junichiro Shiomi, ${ }^{\S}$

Kouhei Takahashi, ${ }^{\ddagger}$ Gordon S. W. Craig, ${ }^{\dagger}$ and Paul F. Nealey $y^{\dagger, *}$

${ }^{\dagger}$ Pritzker School of Molecular Engineering, The University of Chicago, Chicago, Illinois 60637, United States

\$ Technology Innovation Division, Panasonic Corporation, Kyoto 619-0237, Japan

$\S$ Department of Mechanical Engineering, The University of Tokyo, Tokyo 113-8656, Japan 


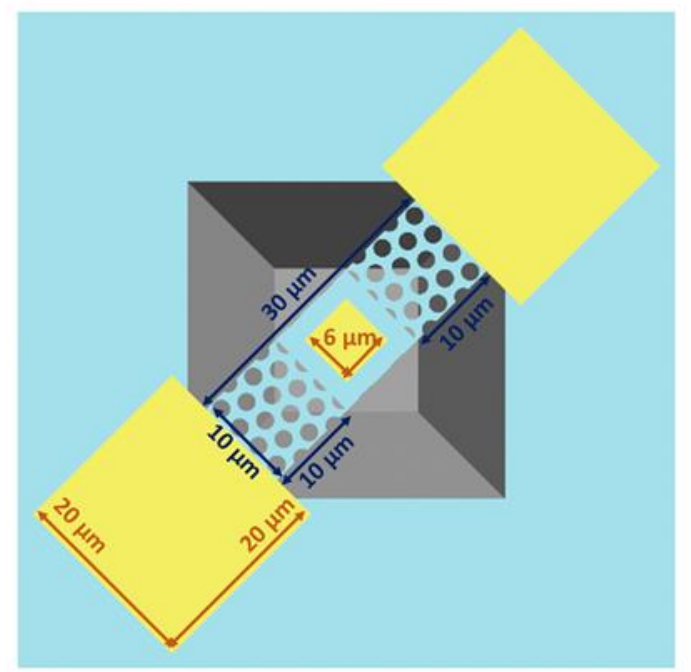

Figure S1. Design of the silicon nitride phononic crystals for thermoreflectance measurement with dimensions labeled.

(a)

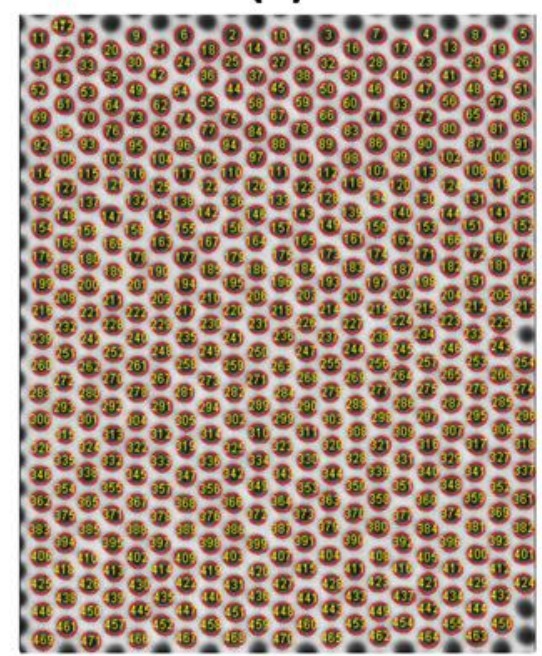

(b)

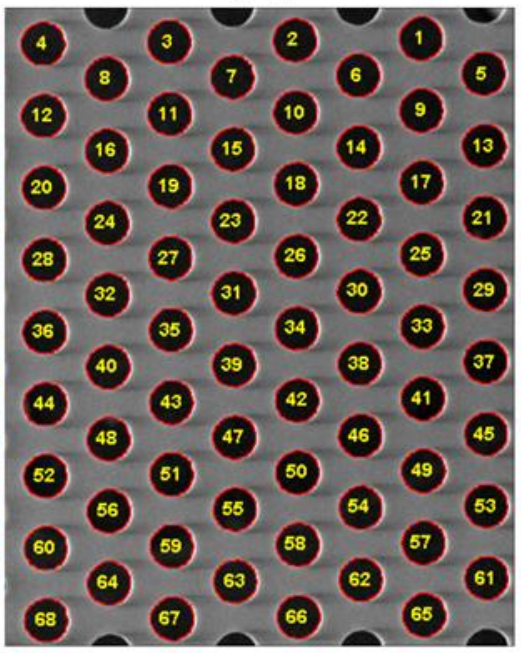

Figure S2. The outlines of (a) DSA and (b) e-beam patterned holes extracted from SEM images. 


\section{Supplementary note 1: Measurement and analysis of thermal transport properties of phononic crystals}

The transient thermal analysis module in ANSYS software was used to calculate $\kappa$ of $\operatorname{SiN}_{x}$ phononic crystals (PnCs) from the measured TDTR signals [Fig. S4(a)]. Assuming that the heat capacity and mass density of $\mathrm{SiN}_{x}$ do not change by nanostructuring, the relaxation times $\tau_{1}$ and $\tau_{2}$, as described in the main text, of the TDTR signals will only depend on $\kappa$ of $\mathrm{SiN}_{x}$. Figure S4(b) shows an example of the measured TDTR signal of an $800 \mathrm{~nm}$ pitch $\mathrm{SiN}_{x} \mathrm{PnC}$ and those simulated by FEM analysis at various values of $\kappa$ of $\mathrm{SiN}_{x}$ in the range of $0.5-4 \mathrm{~W} / \mathrm{mK}$. One can see that the relaxation time of the simulated curves increases with decreasing $\kappa$. This means that $\Delta T$ of the $\mathrm{Al}$ pad is indeed governed by thermal conduction of the $\mathrm{SiN}_{x}$ PnC. Both the simulated and the measured decay curve can be fitted by a sum of two-exponential functions shown in Equation 1 in the main text.

\section{Supplementary note 2: Classical volume reduction effect on thermal conductivity}

The classical effect of volume reduction on the thermal conductivity was calculated by FEM using the steady-state thermal analysis module in ANSYS software. Here, we first calculated the thermal conductance $G$ of a thin rectangular plate with cylindrical through-holes arranged in a triangular lattice replicating our PnC films. A schematic of the three-dimensional model used in our simulation is shown in Fig. S5. In this model, one of the surfaces of the holey film, which we define as surface-A, was fixed at a constant temperature of $T_{\mathrm{A}}\left(=20^{\circ} \mathrm{C}\right)$. A uniform heat flow of $Q$ $(=2 \mu \mathrm{W})$ was applied to surface-B. With the given $\kappa$ of the $\mathrm{SiN}_{x}$ bare film measured by TDTR, the temperature distribution of the holey film in steady state could be calculated by solving the classical heat conduction equation from Fourier's law $\left(\rho C_{\mathrm{p}} \frac{\partial T}{\partial t}=Q+\nabla \cdot(\kappa \nabla T)\right)$ with the ANSYS 
software (see Fig. S6). Using the simulated temperature of surface-B $T_{\mathrm{B}}, G$ of the holey film could be described as

$$
G=Q /\left(T_{\mathrm{B}}-T_{\mathrm{A}}\right)
$$

The predicted thermal conductivity, $\kappa_{\text {predicted, }}$ of the holey $\mathrm{SiN}_{\mathrm{x}}$ film was calculated with

$$
\kappa_{\text {predicted }}=\frac{l}{w t} G
$$

where $w, l$, and $t$ were the width, length, and thickness of the simulated rectangular plate, respectively. The dimensions $w, l$, and $t$ were fixed at $10 \mu \mathrm{m}, 10 \mu \mathrm{m}$, and $70 \mathrm{~nm}$, respectively, to exactly replicate the experimental samples. We carried out the simulations for rectangular plates with a hexagonal array (pitch $=200 \mathrm{~nm}$ ) of holes with fixed values of diameter $(d)$ of $120-144 \mathrm{~nm}$ and porosity $(\phi)$ of $0.33-0.47$. These simulations showed that Fourier's law, which does not include nanoscale size effects, predicts that the magnitude of $\kappa_{\text {predicted }}$ does not depend on $d$ when the porosity $\phi$ of the plate is fixed. The FEM simulations also predicts that $\kappa_{\text {predicted }}$ decreases monotonically with increasing $\phi$ (Figure S7). 


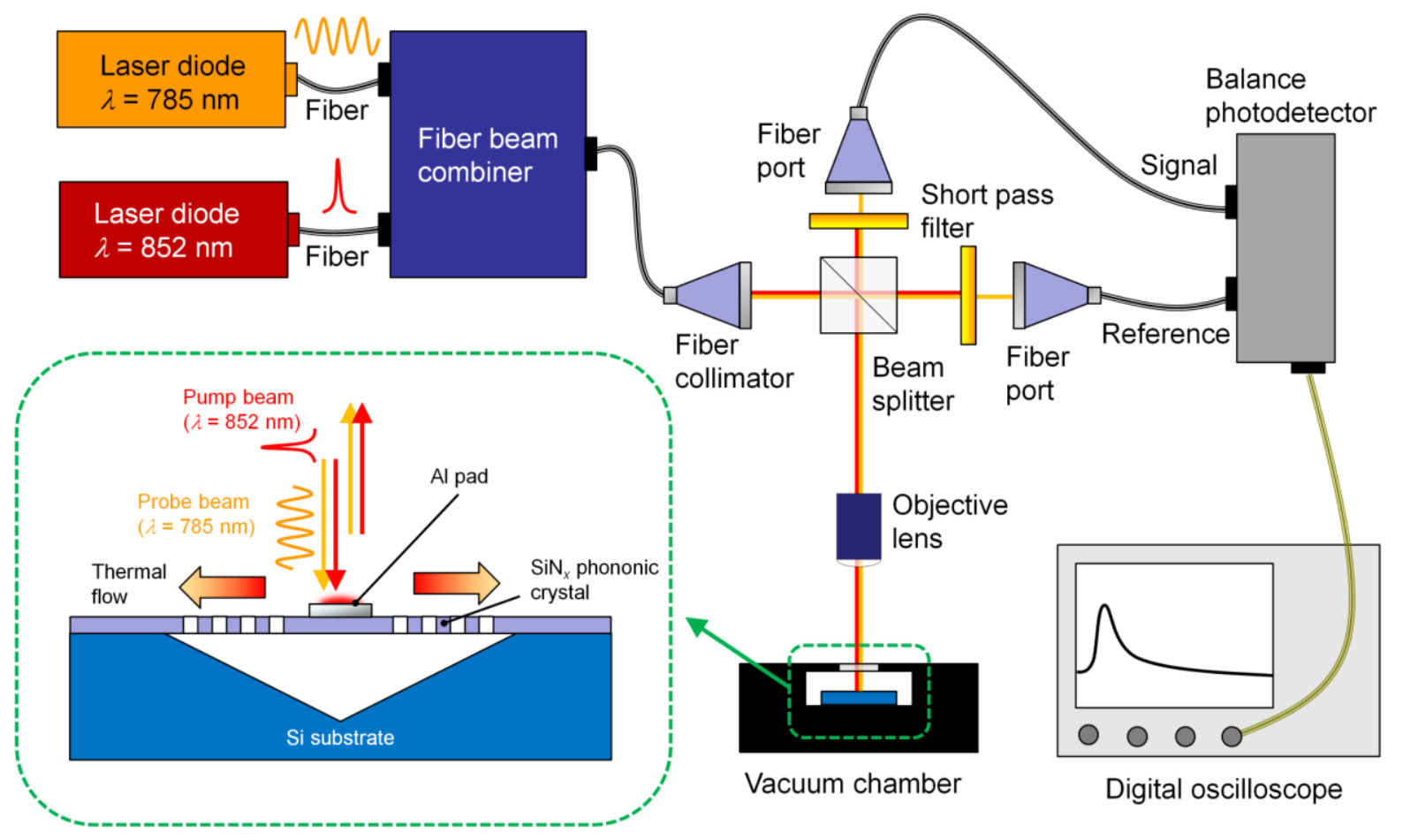

Figure S3. Schematic of the experimental setup of the time-domain thermoreflectance (TDTR) measurement. A typical two-color pump-probe optical system is used to measure the TDTR signal of the Al pad fabricated on top of suspended $\mathrm{SiN}_{x}$ phononic crystal membranes. Perfect parallel alignment of the pump beam and the probe beam is realized by employing a fiber-based system. A schematic cross-section of the sample inside the vacuum chamber is shown in the bottom left corner. 
(a)

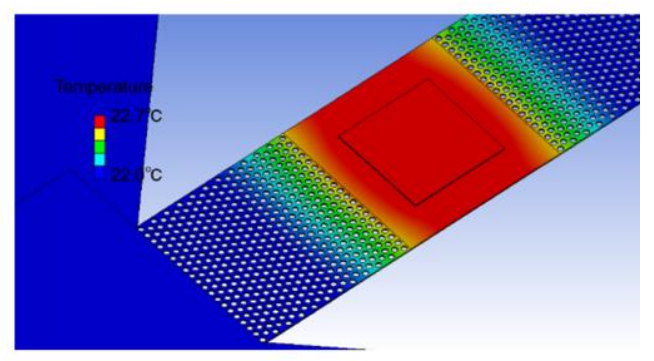

(b)

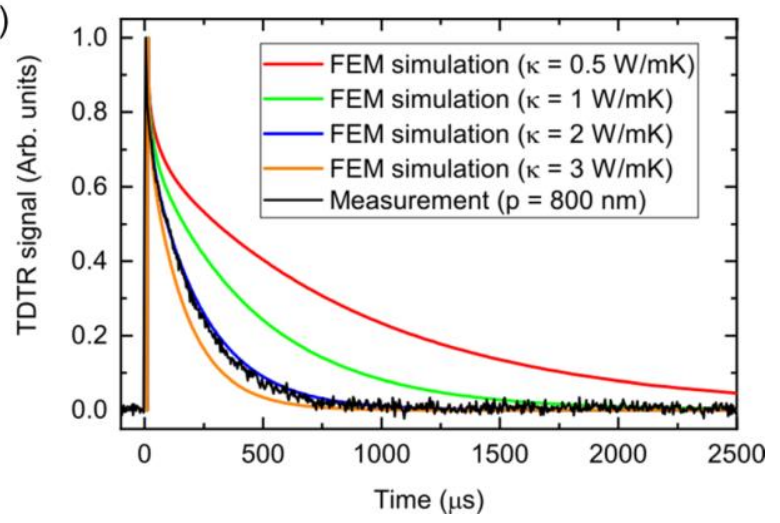

Figure S4. Finite element method (FEM) analysis to determine the thermal conductivity $\kappa$ from the time-domain thermoreflectance (TDTR) measurement. (a) An example of a three-dimensional model of our sample used for the FEM analysis in ANSYS software. (b) Measured TDTR signal of an $800 \mathrm{~nm}$ pitch (p) $\mathrm{SiN}_{x}$ phononic crystal $(\mathrm{PnC})$ and those simulated at various values of material thermal conductivity $(\kappa)$ of $\mathrm{SiN}_{x} \mathrm{PnC}$. 


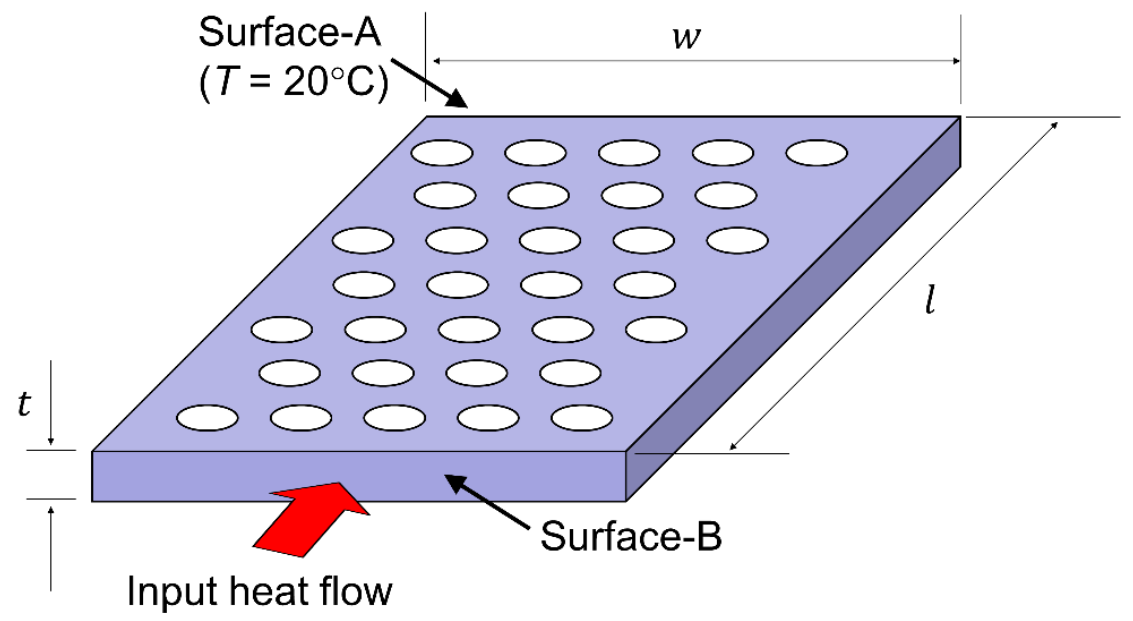

Figure S5. Schematic of the rectangular holey plate replicating a phononic crystal, which was used to examine the classical volume reduction effect on the thermal conductivity. Note that the plate is not drawn to scale. The length $l$, width $w$, and thickness $t$ of the measured samples were used to simulate the thermal conductance of the holey structure. 


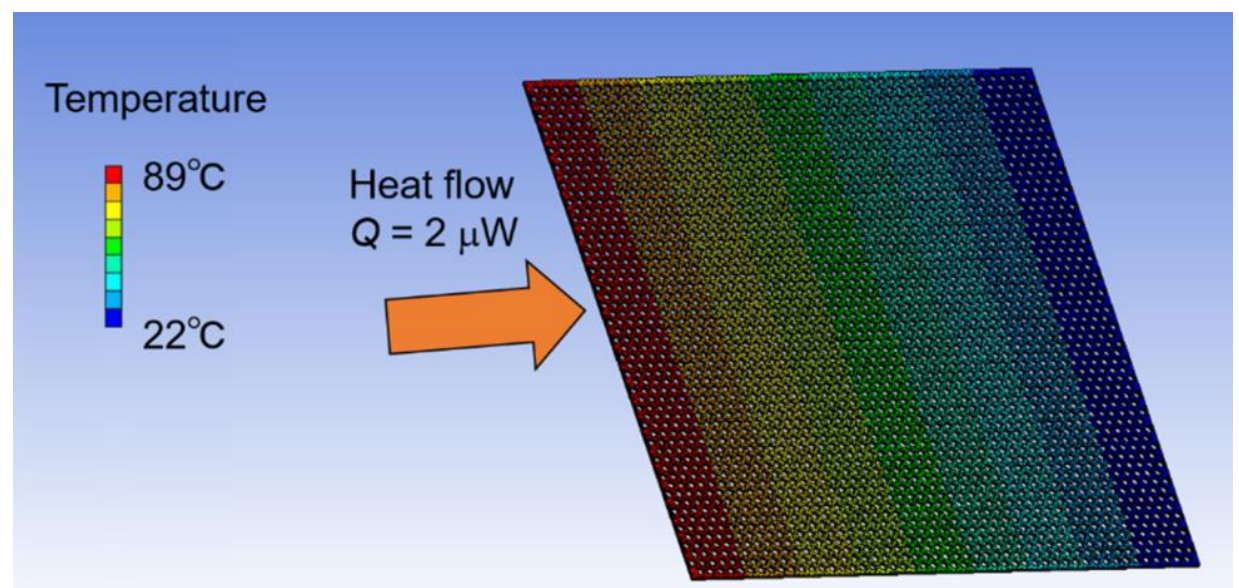

Figure S6. Finite element method model to compute the classical effect of volume reduction on the thermal conductivity. A heat flow $(Q)$ of $2 \mu \mathrm{W}$ was applied on the one surface of thin film. A typical steady state temperature gradient of a SiNx phononic crystals obtained from the simulation is shown as an example. 


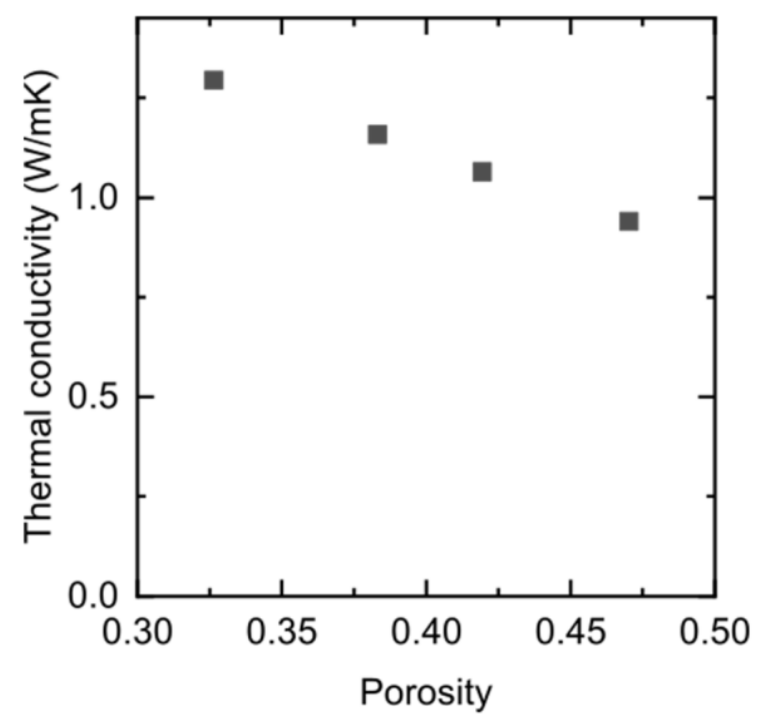

Figure S7. Porosity dependence of the effective thermal conductivity of $\mathrm{SiN}_{x}$ holey films within the scope of the classical Fourier's law. The data points are calculated by finite element method. 